Vol.02/ No. 03

Pages: 146-154

http://irojournals.com/aicn/

DOI: https://doi.org/10.36548/jaicn.2020.3.001

\title{
Tenancy Status Identification of Parking Slots Using Mobile Net Binary Classifier
}

\author{
Dr. Khaled Kamel, \\ Professor, \\ Department of Computer Science, \\ College of Science, \\ Engineering and Technology, \\ Texas Southern University, \\ United States. \\ Email: Khaled.Kamel@tsu.edu

\section{Dr. S. Smys,} \\ Professor, \\ Department of CSE, \\ RVS Technical Campus, \\ Coimbatore, India. \\ Email id: $\underline{\text { smys375@gmail.com }}$ \\ Dr. Abul Bashar, \\ Department of Computer Engineering \\ Prince Mohammad Bin Fahd University \\ Kingdom of Saudi Arabia \\ Email: abashar@pmu.edu.sa
}

\begin{abstract}
The inefficiency in accessing the tenancy status of the parking slots is mainly due to the results of irregular parking regulation/management. The effective parking management enables to avoid unwanted traffic jams and unnecessary fuel wastages. So an efficient parking is necessary for the developing smart cities that aim for a better way of living. So the paper uses the MobileNet Classifier to sort out the tenancy state of the parking slots in the cities to assist a proper parking regulation with better proficiency and perfect management. The Mobile-Net classifiers are a sort of light weight deep neural networks that help in identifying the parking slots available accurately based on the image mined from the live camera that feeds the status of the parking lot continuously. The mechanism put forth detects the patches of images form the live recorded video perfectly and determines the vacant slots. The laid out model was applied in an outdoor parking area to determine the systems effective working on the terms of detection accuracy, false positive and the false negative, true positive and the true negative rates along with the average speed of the in identifying the parking slots. As test case, two different mobile-Net network set up were compared to evaluate the swift ness in processing and the perfectness in detecting.
\end{abstract}

Keywords: Vacant Parking Slots, Tenancy Status, Light Deep Neural Network, Mobile Net Classifiers, Accuracy and Processing Speed.

\section{Introduction}

The day to day rapid increase in the number of vehicles on road has in turn led to the unsurprising increase in the vehicular congestion leading to demands in the parking slots. The continuous increase in the demands on the parking slots has further caused an ugly scenarios in the modern cities that are thriving to become smart. This demand has even resulted in cities "having a hard time taming. Specifically in the busy areas, these "disorganized parking additionally paves way to commuter's woes".

ISSN: 2582-2012 (online)

Submitted: 14.04.2020

Accepted: 01.06.2020

Published: 09.06.2020 
Journal of Artificial Intelligence and Capsule Networks (2020)

Vol.02/ No. 03

Pages: 146-154

http://irojournals.com/aicn/

DOI: https://doi.org/10.36548/jaicn.2020.3.001

Based on the studies appropriately more than five billion of the world's eight billion population is has chosen their living space in the urban areas. This makes the traffic regulation of the cities significant as well as an urgent requisite. But managing the traffic and eluding the congestion to regulate a proper vehicle movement in the city limit is quiet challenging as the proper parking for the vehicles also has to be taken care. At this juncture it is necessary to add this notion, as the world is thriving to construct the smart cities the plan of building or implementing a smart parking system cannot be ignored.

\subsection{Smart Parking}

The latest information's has revealed that the urban motorist spend nearly twenty to thirty minutes on road looking for the a vacant parking slot as the present parking regulation is ineffective. This kind of irregular and improper parking slot management leads to waste of time and fuel creating loss in the "productivity and the economic opportunity. Large amount of fuel gets wasted while searching the parking slot. This paves way for a parking frame work that is integrated. By this the vehicles could identify the proper parking slots even before they enter the premises. This type of system causes considerable saving in the time and the fuel as the slot where the vehicle has to be parked is known before hand

Though multiple applications and digitized solutions has been provided by the researchers in assisting the vehicle drivers find the proper parking slot in the city limit and by collecting and delivering a real time parking information. The developed mobile applications helped in integrating the intelligent transport system with the parking regulation to manage the traffic flow properly. Yet the many conventional system proved to be time consuming and very slow in capturing the information's about the vacant positions once again leading back to poor regulation of parking.

The conventional system faces complexity in understanding the patterns of the vacant slots in the parking spots. In order to have an proper remedy for the problems prevailing in the parking slots and to have a better management of resources many researchers have up with multitude of solutions such as the vision based sensors, "image recognition algorithm with the CNN that delivers a "state of art performances over the challenges incurred in the image net", and many more higher complicated and deeper networks to improve the accuracy and the minimize the cost. Yet the real time tenancy status detection for the parking slots demands for the "image classification chores" to be performed on the embedded resource limited devices.

So the paper uses the Mobile-Net Classifier to sort out the tenancy state of the parking slots in the cities to assist a proper parking regulation with better proficiency and perfect management. The Mobile-Net classifiers are a sort of light weight deep neural networks that help in identifying the parking slots available accurately based on the image mined from the live camera that feeds the status of the parking lot continuously. The mechanism put forth detects the patches of images form the live recorded video perfectly and determines the vacant slots. The laid out model was applied in an outdoor parking area to determine the systems effective working on the terms of detection accuracy, false positive and the false negative, true positive and the true negative rates along with the average speed of the in identifying the parking slots. As test case, two different mobile-Net network set up were compared to evaluate the swift ness in processing and the perfectness in detecting.

The work put forward is arranged with the related works in section 2, proposed Mobile-Net based parking slot identification in section 3 results and discussion in section 4 and conclusion in section 5.

ISSN: 2582-2012 (online) 
Journal of Artificial Intelligence and Capsule Networks (2020)

Vol.02/ No. 03

Pages: 146-154

http://irojournals.com/aicn/

DOI: https://doi.org/10.36548/jaicn.2020.3.001

\section{Related Works}

Many researchers have up with multitude of solutions such as the vision based sensors, "image recognition algorithm with the CNN that delivers a "state of art performances over the challenges incurred in the image net", and many more higher complicated and deeper networks to improve the accuracy and the minimize the cost. The section presents few previous findings to regulate the parking and resolve the problem caused due to improper parking. Suhr et al [1] in his paper has devised sensor fused system to monitor the vacant spaces in the parking. Lee et al [2] integrated the clustering technology in the three sixty degree tracking device to sort out the vacant spaces. Li et al [3] devised a standard, strategy that relies on training to identify the empty space using the machine vision and Zhang et al [4] made changes in the above mentioned system by engaging the deep convolutional neural networks.

Fan et al [5] performed the "Line Filter-Based Parking Slot Detection for Intelligent Parking Assistance System." Scheunert, et al [6] put forth the ""Free space determination for parking slots using a 3D PMD sensor." Li, et al [7] engaged the AVM device to automatically identify the left out empty spaces in the parking areas.Xu, et al [8] conducted the "Vision-guided automatic parking for smart car." Wu et al [9] presents the."PSDet: Efficient and Universal Parking Slot Detection."

Khan et al [10] put forward a Deep-Learning Based Vehicle Count and Free Parking Slot Detection System." and Zinelli, et al [11] devised an "A deep-learning approach for parking slot detection on surround-view images." Ahn et al [12] Duong et al [13] Hong et al [14] and Li et al [15] utilized the light weight based on the cascade- residualparadigm, deep neural networks in the recognizing the faces and detecting the objects specifically for the devices with limited resources availability, the performances of the light weight network was better in the real time object detection. Phan, et al [16] elaborates the utilization of the mobile binary network in distinguishing the images. The architecture of the mobile binary net used in classifying the images is depicted below in the figure. 1

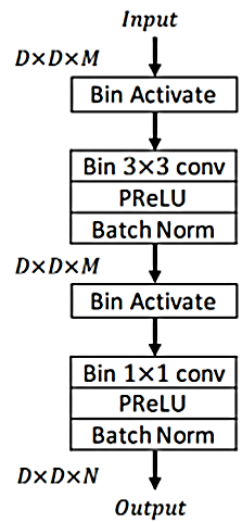

(a) Standard dw-block

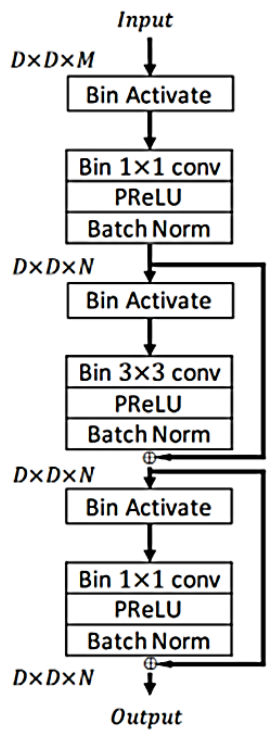

(b) Pre-block

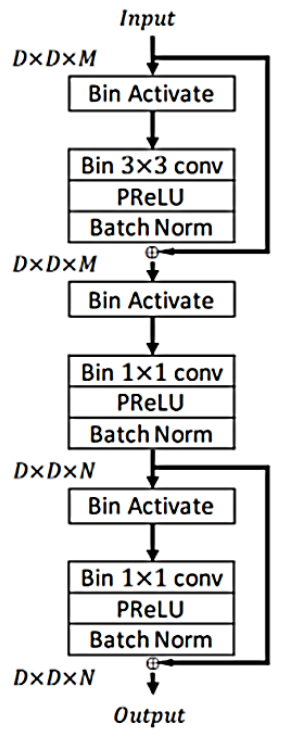

(c) Mid-block

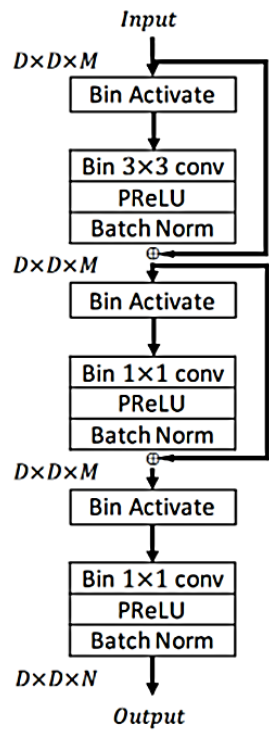

(d) Post-block

Figure.1 MOBILENET Architecture in Image Classification [16]

ISSN: 2582-2012 (online)

Submitted: 14.04.2020

Accepted: 01.06.2020

Published: 09.06.2020 
Journal of Artificial Intelligence and Capsule Networks (2020)

Vol.02/ No. 03

Pages: 146-154

http://irojournals.com/aicn/

DOI: https://doi.org/10.36548/jaicn.2020.3.001

Ilhan,et al [17] devised "A fully automated hybrid human sperm detection and classification system based on mobilenet and the performance comparison with conventional methods."Pandian, M. D. et al [18] put forth the Sleep Pattern Analysis and Improvement Using Artificial Intelligence and Music Therapy. Journal of Artificial Intelligence. Bashar, A. et al [19] devised a "Survey on Evolving Deep Learning Neural Network Architectures. Journal of Artificial Intelligence," Bindhu, Vet al [20] put forth the "Biomedical Image Analysis Using Semantic Segmentation."

Kumar, T. Senthil et al [21] devised the. "A Novel Method for HDR Video Encoding, Compression and Quality Evaluation." Koresh, M. H. J. D et al [22] proposed the "Computer Vision Based Traffic Sign Sensing for Smart Transport" from the papers surveyed using the related works it was found that the deep neural network based architectures demands bigger dimension frame works to attain a adequate small network to run on the platforms with the resource limitations. Providentially the mobile net binary classifiers that are a light weight deep neural networks could hypothetically satisfy the demands of the applications that requites low-latency. The work laid out in the paper puts forward the significance of the MOBILENET classifier in identify the tenancy status of the parking area in the outdoor parking spaces and further provides the "accuracy-speed" trade off various setup of the MOBILENET classifiers and tests the application of the classifiers on identifying the empty spaces in the parking areas for tenancy status identification for empty parking spaces with less delay on the embedded device.

\section{Proposed Work}

The system model is encompassed with three level of process to identify the tenancy status of the parking areas. The figure .2 shows the various unit encompassed in the system for assisting the parking identification.

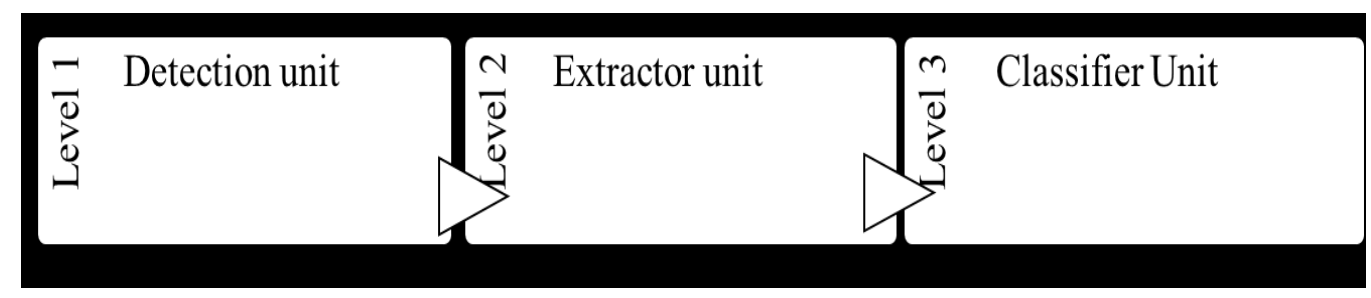

Figure. 2 System Model

The detection unit is comprised with the Internet protocol camera that is capable of receiving regulation information as well as sending images through the internet. These cameras are most commonly engaged in the surveillance, yet unlike closed circuit television cameras they don't demand for a local recording device but for LAN connection to connect to the nearby base station and function as the "detection unit" in the level one process. The figure. 3 depicts the architecture of the proposed parking slot tenancy status detection.

ISSN: 2582-2012 (online) 
Journal of Artificial Intelligence and Capsule Networks (2020)

Vol.02/ No. 03

Pages: 146-154

http://irojournals.com/aicn/

DOI: https://doi.org/10.36548/jaicn.2020.3.001

Extractor Unit

Classifier Unit

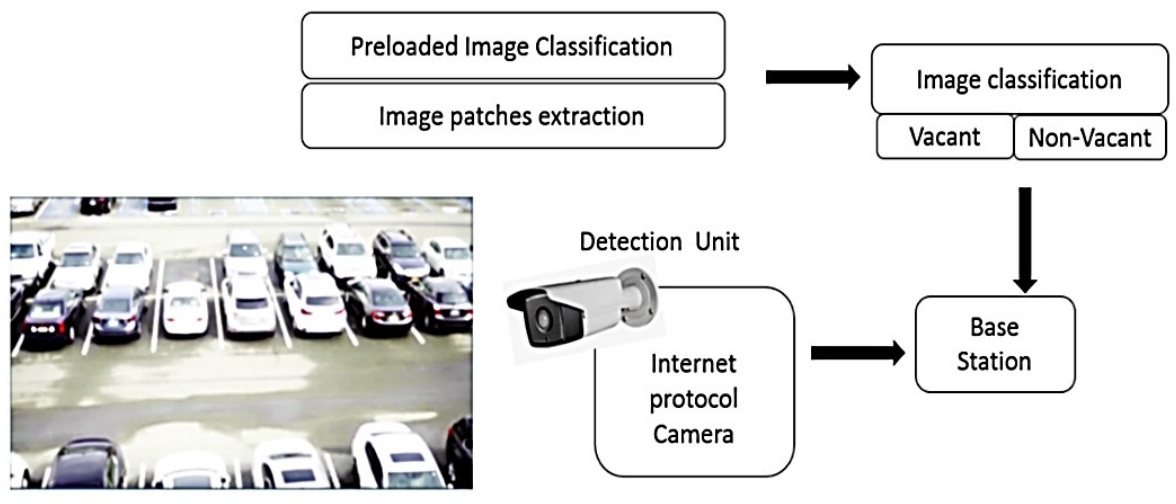

Figure.3 Proposed Architecture

The images gathered with the help of the detection unit is processed in the extractor unit to extricate the patches of the images of the separate spaces in the parking areas according to the co-ordinates of the every parking space specified by the annotation file. The image patches acquired are physically segregated as vacant and non- vacant, and are used in training, the convolutional neural network using the MOBILENET Binary classifier, to acquire a framework trained to distinguish the images. The light weight structure of the MOBILENET Binary classifier makes it compatible for the real time appliances. The output of the classification frame work in the form of "optimally adjusted weight values" is fed into the base station to enable the identification of the vacant spaces in the parking areas.

The MOBILENET Binary classifiers uses the supplementary "point wise convolution" at the three stages following the input, they are the pre-block, Mid-block and the Post-block as shown in figure.1 The block construction heightens the skip connectors allowing the separable layers in the CNN to preserve the original features. The "ReLU is replace with the PReLU to provide the "Convergence Stability." The estimation of flow in every segment of the convolution starts with the "INPUT $\rightarrow$ Binary Activation $\rightarrow$ Binary Convolution $\rightarrow$ PReLU $\rightarrow$ Batch Normalization $\rightarrow$ Output." The pooling layers in the MOBILENET binary classifier in order to gradually shrink the spatial dimensions via network. The system prefers an average pooling to provide better empirical results [16].

The base Station simultaneously repossesses the images of the real lively parking spaces from the cameras fixed and affords to deliver a real time tenancy status identification of the parking slots. For every images gathered the images patches of the vacant and the non-vacant spaces in the parking area are cropped and concurrently the classification between the vacant and the non-vacant is sorted out using the patches of images as input to the MOBILE NET binary classifier that incorporates the "preloaded image segregation model."

\section{Results and Discussion}

\subsection{Implementation}

The system is encompassed with the "FOSCAM FI9800P" internet protocol camera mounted on the lamp posts in the parking area. The training images were gathered on the duration of three to four weeks, 24/7 from an outdoor parking

ISSN: 2582-2012 (online)

150

Submitted: 14.04 .2020

Accepted: 01.06.2020

Published: 09.06.2020 
Journal of Artificial Intelligence and Capsule Networks (2020)

Vol.02/ No. 03

Pages: 146-154

http://irojournals.com/aicn/

DOI: https://doi.org/10.36548/jaicn.2020.3.001

area with 50 layouts. The system uses the "label MG" as the annotating file to define the area of the separate parking areas. The patches of the images of fifty parking space are extricated using the OPENCV and are distinguished as vacant and non-vacant. More than $5 \mathrm{~K}$ real life images are used in training the classifier to yield better results. The tensor flow does the training operation.

The "accuracy-speed" trade off various setup of the MOBILENET binary classifiers (baseline and the minimal) is done by fixing the width and the resolution multiplier to its corresponding minimal and maximal values. A uniform pruning of width multiplier is performed and the resolution multiplier is fixed base on the resolution of the input image fed. The two diverse MOBILENET binary classifiers are trained at the learning rate of $.01 \%$ with the number of iterations $=3000$. The proposed work engages the "ASUS tinker board as base station and the embedded computer with Quad Processor $(1.8 \mathrm{GHz})$ and 2 GB RAM. The DBOS (TINKER) is installed in the Tensor module compatible with the ARM. The MOBILENET Binary classifier is fed as application written in python into the tensor flow frame work. The JSON format is utilized to display the tenancy status of the spaces in the parking area.

\subsection{Result Analysis}

The qualitative analysis of the proposed frame work is performed by determining the performance metric like false positive, false negative, true positive and true negative. Using the performance metric the average accuracy of the detection is determined as shown in equation 1

$$
\text { Detection Accuracy }=\frac{\text { True }_{\text {positive }}+\text { True } \text { negative }}{\text { True positive }+ \text { True } \text { negative }+ \text { False } \text { positive }+ \text { False } \text { Negative }}
$$

The table. 1 below depicts the identification accuracy of the two MOBILENET Binary Classifiers and also present the false positive ratio and the false negative ratio of the proposed model for the testing images acquired through the IP camera for a week.

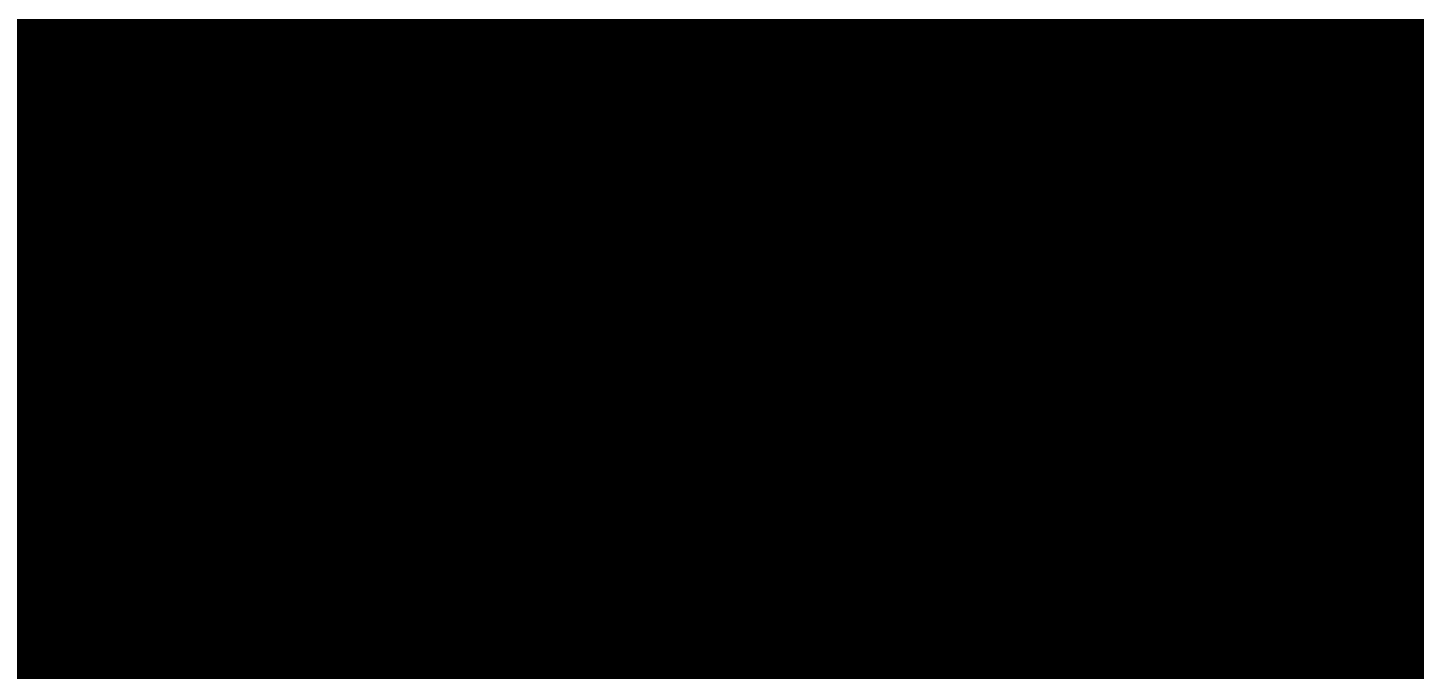

ISSN: 2582-2012 (online)

Submitted: 14.04 .2020

Accepted: 01.06.2020

Published: 09.06.2020 
Journal of Artificial Intelligence and Capsule Networks (2020)

Vol.02/ No. 03

Pages: 146-154

http://irojournals.com/aicn/

DOI: https://doi.org/10.36548/jaicn.2020.3.001

Table.1 Identification Accuracy, False Positive Rate, False Negative Rate

The figure. represents the time taken by the two diverse MOBILENET Binary Classifiers to classify the patches of images. The proposed model affords to deliver the tenancy status within 2.0 seconds.

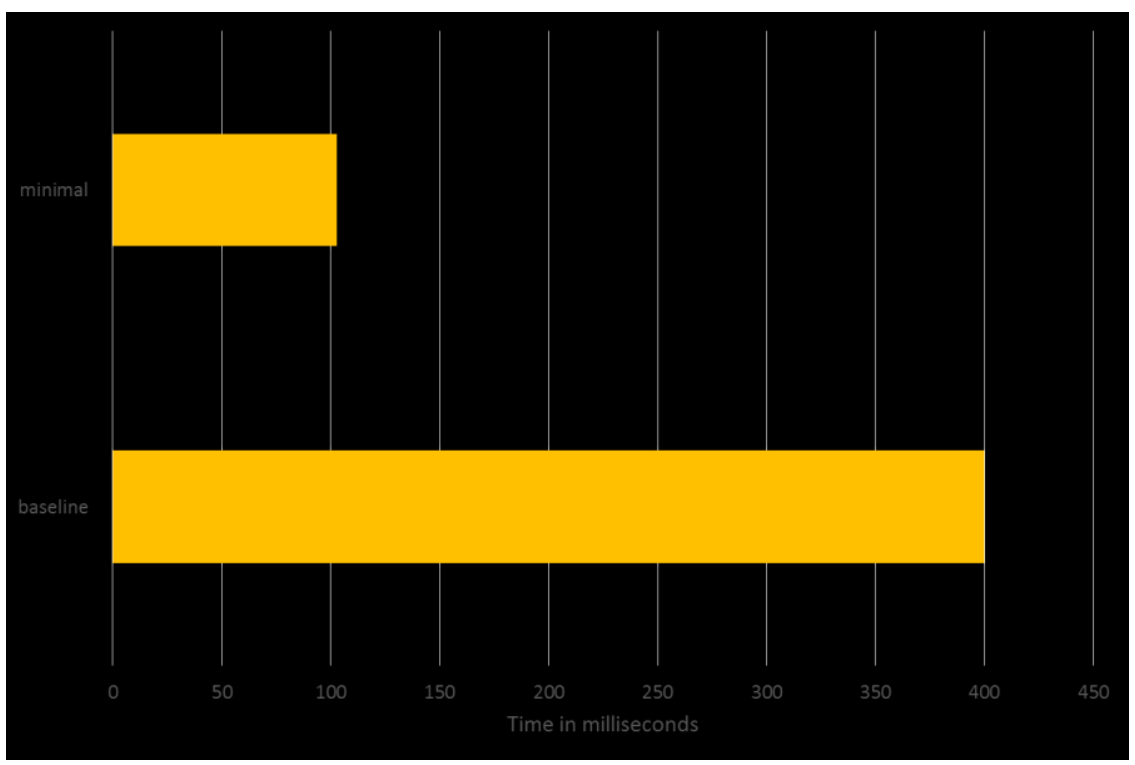

Figure.4 Time Utilized.

As a summary from the results observed minimal MOBILENET Binary Classifiers proves be the choicest frame work for reflecting the results of identification incurring a less delay, without compromising ion the accuracy in identification, the result observed in table 1 shows the accuracy of the same. So the frame put forward in the paper is capable of delivering accurate results with speedy processing. Moreover the proposed system affords to deliver low cost solutions. Compared to other standard approaches. Unlike convolutional neural network method, the MOBILE NET Binary classifier does not demand a manual network customization.

\section{Conclusion}

The effective parking management enables to avoid unwanted traffic jams and unnecessary fuel wastages. So an efficient parking is necessary for the developing smart cities that aim for a better way of living. So the paper uses the Mobile-Net Classifier to sort out the tenancy state of the parking slots in the cities to assist a proper parking regulation with better proficiency and perfect management. The Mobile-Net classifiers are a sort of light weight deep neural networks that help in identifying the parking slots available accurately based on the image mined from the live camera that feeds the status of the parking lot continuously. The mechanism put forth detects the patches of images form the live recorded video perfectly and determines the vacant slots. The laid out model was applied in an outdoor parking area to determine the systems effective working on the terms of detection accuracy, false positive and the false negative, true positive and the true negative rates along with the average speed of the in identifying the parking slots.

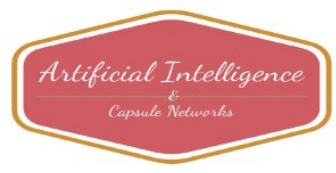


Journal of Artificial Intelligence and Capsule Networks (2020)

Vol.02/ No. 03

Pages: 146-154

http://irojournals.com/aicn/

DOI: https://doi.org/10.36548/jaicn.2020.3.001

Based on the evaluation of "accuracy-speed" trade off various setup of the MOBILENET binary classifiers (baseline and the minimal) the proposed system proved to deliver a low cost solution with the better accuracy and speedy processing.

\section{References}

[1] Suhr, Jae Kyu, and Ho Gi Jung. "Sensor fusion-based vacant parking slot detection and tracking." IEEE Transactions on Intelligent Transportation Systems 15, no. 1 (2013): 21-36.

[2] Lee, Soomok, Daejin Hyeon, Gikwang Park, Il-joo Baek, Seong-Woo Kim, and Seung-Woo Seo. "Directional-DBSCAN: Parking-slot detection using a clustering method in around-view monitoring system." In 2016 IEEE Intelligent Vehicles Symposium (IV), pp. 349-354. IEEE, 2016.

[3] Li, Linshen, Lin Zhang, Xiyuan Li, Xiao Liu, Ying Shen, and Lu Xiong. "Vision-based parking-slot detection: A benchmark and a learning-based approach." In 2017 IEEE International Conference on Multimedia and Expo (ICME), pp. 649-654. IEEE, 2017.

[4] Zhang, Lin, Junhao Huang, Xiyuan Li, and Lu Xiong. "Vision-based parking-slot detection: A DCNNbased approach and a large-scale benchmark dataset." IEEE Transactions on Image Processing 27, no. 11 (2018): 5350-5364.

[5] Fan, Mengyang, Zhencheng Hu, Kazukuni Hamada, and Hui Chen. "Line Filter-Based Parking Slot Detection for Intelligent Parking Assistance System." In Proceedings of SAE-China Congress 2014: Selected Papers, pp. 175-181. Springer, Berlin, Heidelberg, 2015.

[6] Scheunert, Ullrich, Basel Fardi, Norman Mattern, Gerd Wanielik, and Norbert Keppeler. "Free space determination for parking slots using a 3D PMD sensor." In 2007 IEEE Intelligent Vehicles Symposium, pp. 154-159. IEEE, 2007.

[7] Li, Lei, Changle Li, Qieshi Zhang, Tao Guo, and Zhifang Miao. "Automatic parking slot detection based on around view monitor (AVM) systems." In 2017 9th International Conference on Wireless Communications and Signal Processing (WCSP), pp. 1-6. IEEE, 2017.

[8] Xu, Jin, Guang Chen, and Ming Xie. "Vision-guided automatic parking for smart car." In Proceedings of the IEEE Intelligent Vehicles Symposium 2000 (Cat. No. 00TH8511), pp. 725-730. IEEE, 2000.

[9] Wu, Zizhang, Weiwei Sun, Man Wang, Xiaoquan Wang, Lizhu Ding, and Fan Wang. "PSDet: Efficient and Universal Parking Slot Detection." arXiv preprint arXiv:2005.05528 (2020).

[10] Khan, Gulraiz, Muhammad Ali Farooq, Zeeshan Tariq, and Muhammad Usman Ghani Khan. "DeepLearning Based Vehicle Count and Free Parking Slot Detection System." In 2019 22nd International Multitopic Conference (INMIC), pp. 1-7. IEEE, 2019.

[11] Zinelli, Andrea, Luigi Musto, and Fabio Pizzati. "A deep-learning approach for parking slot detection on surround-view images." In 2019 IEEE Intelligent Vehicles Symposium (IV), pp. 683-688. IEEE, 2019.

[12] Ahn, Namhyuk, Byungkon Kang, and Kyung-Ah Sohn. "Fast, accurate, and lightweight super-resolution with cascading residual network." In Proceedings of the European Conference on Computer Vision (ECCV), pp. 252-268. 2018.

[13] Duong, Chi Nhan, Kha Gia Quach, Ibsa Jalata, Ngan Le, and Khoa Luu. "Mobiface: A lightweight deep learning face recognition on mobile devices." arXiv preprint arXiv:1811.11080 (2018).

[14] Hong, Sanghoon, Byungseok Roh, Kye-Hyeon Kim, Yeongjae Cheon, and Minje Park. "PVANet: Lightweight deep neural networks for real-time object detection." arXiv preprint arXiv:1611.08588 (2016).

[15] Li, Yuxi, Jiuwei Li, Weiyao Lin, and Jianguo Li. "Tiny-dsod: Lightweight object detection for resourcerestricted usages." arXiv preprint arXiv:1807.11013 (2018).

[16] Phan, Hai, Yihui He, Marios Savvides, and Zhiqiang Shen. "Mobinet: A mobile binary network for image classification." In The IEEE Winter Conference on Applications of Computer Vision, pp. 34533462. 2020.

ISSN: 2582-2012 (online)

Submitted: 14.04.2020

Accepted: 01.06.2020

Published: 09.06.2020 
Journal of Artificial Intelligence and Capsule Networks (2020)

Vol.02/ No. 03

Pages: 146-154

http://irojournals.com/aicn/

DOI: https://doi.org/10.36548/jaicn.2020.3.001

[17] Ilhan, Hamza O., I. Onur Sigirci, Gorkem Serbes, and Nizamettin Aydin. "A fully automated hybrid human sperm detection and classification system based on mobile-net and the performance comparison with conventional methods." Medical \& Biological Engineering \& Computing (2020): 1-22.

[18] Pandian, M. D. (2019). Sleep Pattern Analysis and Improvement Using Artificial Intelligence and Music Therapy. Journal of Artificial Intelligence, 1(02), 54-62.

[19] Bashar, A. (2019). Survey on Evolving Deep Learning Neural Network Architectures. Journal of Artificial Intelligence, 1(02), 73-82.

[20] Bindhu, V. "Biomedical Image Analysis Using Semantic Segmentation." Journal of Innovative Image Processing (JIIP) 1, no. 02 (2019): 91-101.

[21] Kumar, T. Senthil. "A Novel Method for HDR Video Encoding, Compression and Quality Evaluation." Journal of Innovative Image Processing (JIIP) 1, no. 02 (2019): 71-80.

[22] Koresh, M. H. J. D. (2019). Computer Vision Based Traffic Sign Sensing For Smart Transport. Journal of Innovative Image Processing (JIIP), 1(01), 11-19

\section{Authors Biography}

Dr. Khaled A. Kamel is currently Chairman and Professor at Texas Southern university, College of Science and technology, Department of Computer Science, Houston, TX. He has published many research articles in refereed journals and IEEE conferences. He has more than 30 years of teaching and research experience. He has been the General chair, Session Chair, TPC Chair and Panelist in several conferences and acted as a reviewer and guest editor in referred journals. His research interest includes Networks, Computing, and Communication systems.

Dr. S. Smys received his M.E and Ph.D degrees all in Wireless Communication and Networking from Anna University and Karunya University, India. His main area of research activity is localization and routing architecture in wireless networks. He serves as Associate Editor of Computers and Electrical Engineering (C\&EE) Journal, Elsevier and Guest Editor of MONET Journal, Springer. He is served as a reviewer for IET, Springer, Inderscience and Elsevier journals. He has published many research articles in refereed journals and IEEE conferences. He has been the General chair, Session Chair, TPC Chair and Panelist in several conferences. He is member of IEEE and senior member of IACSIT wireless research group. He has been serving as Organizing Chair and Program Chair of several International conferences, and in the Program Committees of several International conferences. Currently he is working as professor in department of comuter science in RVS technical campus, in Tamil nadu India

Dr. AbulBashar is currently working as Assistant Professor at Prince Mohammad Bin Fahd University, Kingdom of Saudi Arabia in the College of Computer Engineering and Sciences. Earlier, he completed his PhD from the School of Computing and Information Engineering at the University of Ulster, Coleraine, UK in 2011. He received his B.E. degree in Electronics \& Communication Engineering from Osmania University, Hyderabad, India in 1995. He has an M.S. degree in Electrical Engineering from King Fahd University of Petroleum \& Minerals (K.F.U.P.M.), Dhahran, Kingdom of Saudi Arabia in 1999. Before joining his PhD research he was a Lecturer for 8 years in the Electrical Engineering department at K.F.U.P.M. He is a recipient of Osmania University Engineering Gold Medal in 1995, M.S. Research Scholarship from KFUPM (1996) and Vice Chancellors Research Scholarship from University of Ulster (2008). He is actively involved in the TPC/Review

ISSN: 2582-2012 (online)

Submitted: 14.04.2020

Accepted: 01.06.2020

Published: 09.06.2020 
Journal of Artificial Intelligence and Capsule Networks (2020)

Vol.02/ No. 03

Pages: 146-154

http://irojournals.com/aicn/

DOI: https://doi.org/10.36548/jaicn.2020.3.001

committees of renowned journals and conferences namely CSC IJCN, IEEE WCNC 2011, ISCI 2011, ICDIPC 2011, ICSECS 2011, DICTAP 2011 and NDT 2009/2010/2012

ISSN: 2582-2012 (online)

Submitted: 14.04 .2020

Accepted: 01.06.2020

Published: 09.06.2020 